\title{
"Essential Tremor Plus": A Problematic Concept: Implications for Clinical and Epidemiological Studies of Essential Tremor
}

\author{
Elan D. Louis ${ }^{\mathrm{a}-\mathrm{c}}$ \\ a Division of Movement Disorders, Department of Neurology, Yale School of Medicine, Yale University, New Haven, CT, USA; \\ ${ }^{b}$ Center for Neuroepidemiology and Clinical Neurological Research, Yale School of Medicine, Yale University, New Haven, \\ CT, USA; ' Department of Chronic Disease Epidemiology, Yale School of Public Health, Yale University, New Haven, CT, USA
}

\section{Keywords \\ Essential tremor $\cdot$ Epidemiology $\cdot$ Nomenclature $\cdot$ Case \\ definition - Essential tremor-plus}

\begin{abstract}
Background: An issue of central importance for most neurological disorders is that of case definition. In the field of neuroepidemiology, case definition informs the design, conduct, and interpretation of research studies that range from observational to interventional studies, from case-control to cohort studies, and from descriptive to analytical studies. For essential tremor (ET), one of the most common neurological disorders, the issue of case definition is particularly important. Though traditionally viewed as a monochromatic disorder, recent years have seen an expansion of the clinical phenotype. In a recently proposed consensus statement on tremor, a proposal was made to separate from ET a new tentatively and uncertainly-defined entity characterized by the presence of additional neurological signs other than action tremor. This entity would be termed "ET-plus". The aims of this invited paper are to discuss the impact of the proposed nomenclature on studies of ET neuroepidemiology. Summary: The proposed term "ET-plus" is based solely on clinical
\end{abstract}

๑) 2020 S. Karger AG, Basel features and even these are not clearly defined. It is not based on differences in underlying etiology (e.g., genes) or pathology. As such, it is not grounded in underlying biological differences. The use of the term would have numerous research implications. As detailed here, it would greatly complicate or make impossible studies of the descriptive epidemiology of ET, association studies, cohort studies, and clinical trials in ET. Key Messages: The proposed designation has the potential to be very problematic for studies of the epidemiology of tremor. This, along with this questionable validity of the proposed nomenclature, calls its use into serious question.

(c) 2020 S. Karger AG, Basel

\section{Introduction}

Introduction to the Issue of Case Definition

An issue of central importance for most neurological disorders is that of case definition. How a case is defined has numerous implications, not only clinically but in terms of scientific research. For the field of neuroepidemiology, this is particularly true. Ironically, neurological disorders are often challenging to define, as they are often

\section{KARGER}

karger@karger.com

www.karger.com/ned
Elan D. Louis

Division of Movement Disorders, Department of Neurology

Yale School of Medicine, Yale University

15 York Street, New Haven, CT 06520-8018 (USA)

E-Mail elan.louis@yale.edu 
phenotypically complex and characterized by a phenomenology that is changing and evolving with time. All the same, how cases are defined affects the design, conduct, and interpretation of the full panoply of neuroepidemiological research studies, ranging from observational to interventional studies, from case-control to cohort studies, and from descriptive to analytical studies.

For essential tremor (ET), one of the most common neurological diseases, the issue of case definition is particularly important $[1,2]$. Though traditionally viewed as a more or less monochromatic disorder, ET has also been increasingly difficult to fully encapsulate. As a result, numerous definitions, some of them quite divergent, have arisen over the years [3-5].

\section{Recent Trends in the Understanding of ET}

In recent years, it has become increasingly clear that the notion that ET is a mono-symptomatic condition, characterized solely by isolated upper limb action tremor, is an outdated and over-simplified one. As with other chronic, progressive, late-life neurological diseases such as Parkinson's disease and Huntington's disease, the core motor feature seems to be accompanied by additional motor features as well as a range of non-motor features. This point merits further elaboration and contextualization.

First, it has long been thought that the patho-mechanistic basis of ET is an aberrant cerebello-thalamo-cortical loop [6-14], and postmortem evidence has increasingly identified structural, degenerative changes in the ET cerebellum itself [15-28]. Given this pathological anatomy, it would not be surprising that mild gait ataxia may accompany ET [29-32]. Similarly, although dystonia was originally viewed as a disorder of the basal ganglia, recent evidence indicates a role of the cerebellum $[33,34]$. As such, it is conceivable that in a disorder such as ET, which involves the cerebellum, there may be some dystonia.

Second, as the disease progresses, kinetic tremor in ET worsens $[35,36]$. Additionally, with time, patients often experience the successive addition of tremors that occur under different activation conditions (e.g., with intention [37], at rest $[38,39]$ ) and in different body regions (e.g., neck, jaw, or voice) [40,41]. The presence of these tremors, as well as the presence of gait and balance difficulty, is associated with ET of longer duration [37, 39, 41, 42]. That is, ET patients accumulate these additional clinical features with the passage of time [43].

Third, clinical and epidemiological studies have demonstrated that ET patients have an increased odds and/or risk of developing both mild cognitive impairment and dementia, and that milder cognitive issues are more prevalent in ET cases than controls [44-47]. There is a cognitive side to ET [48] and other non-motor features, some of which are psychiatric [49] and some of which are sensory [50], are apparent.

\section{Recently Proposed Nomenclature}

In a recent consensus statement on tremor, written by a panel of experts for the International Parkinson and Movement Disorder Society, a proposal was made to separate from ET a new tentatively and uncertainly-defined entity characterized by the presence of additional neurological signs other than action tremor [51]. This entity would be termed "ET-plus". Thus, in the Consensus statement [51], ET was defined as (1) isolated tremor syndrome of bilateral upper limb action tremor, (2) at least 3 years' duration, (3) with or without tremor in other locations (e.g., head, voice, or lower limbs), and (4) absence of other neurological signs, such as dystonia, ataxia, or parkinsonism [51]. ET-plus was defined as tremor with the characteristics of ET and additional neurological signs of uncertain significance such as impaired tandem gait, questionable dystonic posturing, memory impairment, or other mild neurological signs of unknown significance that do not suffice to make an additional syndrome classification or diagnosis. ET with tremor at rest would be classified as ET-Plus [51].

The proposed nomenclature has already engendered controversy $[52,53]$. It is now the focus of a tide of papers, and numerically, one may envision the potential impact of this nomenclature - a study recently reported that with the use of the proposed nomenclature, 110 (83\%) of 133 ET cases would have to be re-classified as ET-plus [54]. In another study, 99 (39.3\%) of $252 \mathrm{ET}$ cases would have to be re-classified as such [53]. In some circles, the nomenclature is already being adopted $[55,56]$.

It is important to point out that the term "ET-plus" is based solely on clinical grounds. It is not based on a difference in underlying etiology (i.e., a gene or a certain set of genes), pathology, or an appreciable difference in prognosis. Thus, the proposed term does not have an underlying biological basis.

\section{Aims of the Current Paper}

The aims of the current paper are to discuss the relevance and potential impact of the proposed nomenclature on the field of ET neuroepidemiology. The impact is potentially considerable; hence, it requires some elaboration, reflection, and discussion. 


\section{Methods}

The use of the term "ET-Plus" has numerous potential implications for clinical and epidemiological studies of ET. Before these are discussed, we provide a general discussion of the issue of case definition in ET. We then discuss the numerous potential implications of the proposed nomenclature in the series of subsections that follow.

\section{General Discussion of the Issue of Case Definition in ET}

As noted above, for ET, the issue of case definition is particularly important $[1,2]$. Despite this, defining ET is challenging. There are a number of reasons for this. Action tremor, which is the core motor feature of ET, can be a feature of numerous other neurological disorders and can be a feature of normal aging [57]. The absence of an in vivo biological marker or postmortem pathological diagnostic criteria further create difficulties for those who wish to define ET by means other than clinical. The presence of clinical heterogeneity also makes it challenging to neatly encapsulate everything under one rubric, and the long duration of disease and evolution of phenotype further challenge these attempts [41]. The phenotypic overlap with other neurological disorders, especially dystonia, creates additional challenges $[2,58]$. These have been longstanding issues and they will continue to create challenges. For this reason, new nomenclature should be carefully considered so as not to create more difficulties.

\section{Problems Created for Studies of Descriptive Epidemiology of ET}

The use of the new nomenclature would unnecessarily complicate incidence studies of ET. For example, when performing a study of the incidence of ET-plus, would an investigator be obligated to exclude individuals with baseline ET, because they already have some form of ET, or would one include them because at baseline they did not have the precise outcome of interest (i.e., ETplus)?

The proposed nomenclature would also add to the burden and expense of prevalence studies of ET. Most prevalence studies of ET involve an initial screening phase followed by a more detailed phase, which includes a neurological examination. If one were to adopt the proposed nomenclature, the presence of dystonia, rest tremor, ataxia, or cognitive difficulty would preclude the ET diagnosis; therefore, investigators would have to administer additional questionnaires and perform a more detailed neurological and neuropsychological examination to properly assess these items. The incorporation of an examination of cognition into prevalence studies would perhaps most of all add to the burden.

The proposed nomenclature creates numerous ill-defined boundaries, thereby creating operational questions. Which test or test batteries would be the minimum ones that an investigator would need to employ to properly assess cognition? Would a screening survey, such as the Montreal Cognitive Assessment, be adequate, or would cognition need to be assessed with less superficiality, and in its numerous dimensions and domains (executive function, visuospatial function, memory, etc)? Furthermore, how would one account for the effects of normal aging on cognition with aging alone, there is a decline in cognitive performance and age-associated norms are established for most tests. How much "cognitive impairment" would need to be present to exclude someone as having ET?
The proposed scheme creates additional boundary issues. Thus, the presence of "questionable dystonia" is a proposed exclusionary criterion for ET. What is "questionable dystonia"? Does this mean the examiner is undecided as to whether it is indeed present? Does it mean the phenomenology is ambiguous? Does it simply mean mild dystonia? If that were the case, would it be better to qualify the word "dystonia" with the word "mild"? How much dystonia would be too much for ET?

One further boundary issue is what is meant by "impaired tandem gait"? When testing tandem gait, the number of steps to the side normally increases with advancing age. Unfortunately, ageassociated norms are for the most part wanting in the literature. How many steps to the side during tandem gait are considered "impaired"? One would need to carefully specify the number of such steps by age stratum.

One final issue is how investigators are expected to compare current results with all of the past results of the prevalence studies? One would have to add all of the "ET" and "ET-plus" together in order to compare that prevalence value to the reported prevalence of "ET" from prior studies?

\section{Problems Created for Association Studies (e.g.,}

Case-Control Studies)

The adoption of the ET-plus nomenclature would complicate association studies. For example, how would one examine the association between ET and dementia or between ET and Parkinson's disease, if the presence of the associated condition (i.e., dementia or Parkinson's disease) dis-allowed the ET diagnosis itself? In fact, the adoption of the proposed nomenclature would pre-determine the very outcome of such studies, as by definition, ET itself could not be associated with Parkinson's' disease or dementia. Adoption of the proposed nomenclature would make such studies impossible - nomenclature would be limiting science.

\section{Problems Created for Cohort Studies of ET}

The use of the proposed nomenclature would have additional effects. Longitudinal prospective cohort studies will not be possible, as the baseline condition, ET, would in many cases simply disappear during the course of follow-up. Other problems could arise. If one enrolled an ET case who at baseline had rest tremor (hence, necessitating the label "ET-plus"), but at the next assessment did not on neurological examination display this often-transient neurological finding, would one have to change the diagnosis at the next assessment from ET-plus back to ET? This would suggest that ET-plus diagnoses are reversible, evanescent, and unstable.

Another type of cohort study, survival studies, would also become complicated. Cognitive impairment and gait ataxia were noted to be risk factors for mortality among elders with ET [59]. However, were one to adopt the proposed nomenclature, then the presence of each of these risk factors, cognitive impairment and gait ataxia, would dis-allow the diagnosis of ET. In other words, how does one study risk factors for mortality when the presence of the risk factors themselves negates the ET diagnosis?

\section{Problems for Genetic Studies}

The proposed nomenclature would create difficulties for genetic studies. The presence of phenotypic heterogeneity within families (e.g., presence of rest tremor in one relative but not in oth- 
ers or the presence of gait ataxia in one relative but not others) would necessitate that there are 2 neurological conditions within that family rather than one neurological condition with variable clinical expression. From an operational standpoint, if in a family of 10 individuals with ET, one family member also has rest tremor, does this entire family, and all of its associated data, change from an "ET" family to an "ET-plus" family or a "mixed ET and ET plus family"? For a study of ET genetics, would this family no longer be usable?

Problems Created for Interventional Epidemiological Studies of ET (i.e., Clinical Trials)

The ET-plus designation would unnecessarily complicate experimental therapeutic studies. Given the fact that the ET-plus versus ET distinction is not based on any identified biological difference, it is not likely that the 2 proposed diagnostic designations will respond differently to medications. If, however, all trials moving forward must separate ET from ET-plus, then one would have to run 2 parallel sets of trials. Adequate patient recruitment, already a challenging issue in ET trials, would become even more problematic, as the number of available patients for each study would be a fraction of what it is now.

\section{Discussion and Conclusions}

The study of neurological disorders involves the anal$y$ sis of the distribution of those disorders within populations and the identification of risk factors for and conditions associated with those disorders. This is the centerpiece of neuroepidemiology. How one frames and demarcates those conditions is not a matter of minor importance. Hence, considerations of terminology should not be approached lightly or without consideration of their full impact. These issues should be considered carefully and in detail.

The design and interpretation of studies of the epidemiology of ET, one of the most common neurological disorders, are impacted upon by the way the condition is defined. One may go one step further. The results of studies of the epidemiology of ET are predicated on this issue.

A new diagnostic scheme has been proposed for ET. The validity of the nomenclature is questionable. The term "ET-plus" is based solely on clinical grounds. It is not based on etiological differences or biological differences. The proposed scheme has the potential to interfere with the conduct of numerous studies of the epidemiology of ET, ranging from descriptive to analytical, cross-sectional to longitudinal, observational to interventional. Hence, its potential effects would be far-reaching. That the new classification proposed for ET is problematic is in some ways not hugely surprising. If one learns from other examples, consensus re-classifications for other movement disorders (e.g., for dystonia) have been fraught with problems and are considered compromised [60].

The proposal of new terms is easy. Their adoption is often uncritical. Their impact is often unforeseen. To the cautious, it is important to fully consider both the merit and the impact of such terminology. The proposed designation, ET-plus, has the potential to be very problematic for studies of the epidemiology of tremor. This, along with this questionable validity of the proposed nomenclature, calls its use into serious question.

\section{References}

1 Louis ED, Ottman R, Clark LN. Clinical classification of borderline cases in the family study of essential tremor: an analysis of phenotypic features. Tremor Other Hyperkinet Mov (N Y). 2014 Feb;4:220.

2 Louis ED. The evolving definition of essential tremor: What are we dealing with? Parkinsonism Relat Disord. 2018 Jan;46 Suppl 1: S87-91.

3 Bain P, Brin M, Deuschl G, Elble R, Jankovic $J$, Findley L, et al. Criteria for the diagnosis of essential tremor. Neurology. 2000;54(11 Suppl 4):S7.

4 Elble RJ. Diagnostic criteria for essential tremor and differential diagnosis. Neurology. 2000;54(11 Suppl 4):S2-6.

5 Deuschl G, Bain P, Brin M; Ad Hoc Scientific Committee. Consensus statement of the Movement Disorder Society on Tremor. Mov Disord. 1998;13(S3 Suppl 3):2-23.
6 Gövert F, Becktepe JS, Deuschl G. Current concepts of essential tremor. Rev Neurol (Paris). 2016 Aug - Sep;172(8-9):416-22.

7 Helmich RC, Toni I, Deuschl G, Bloem BR. The pathophysiology of essential tremor and Parkinson's tremor. Curr Neurol Neurosci Rep. 2013 Sep;13(9):378.

8 Muthuraman M, Raethjen J, Koirala N, Anwar AR, Mideksa KG, Elble R, et al. Cerebellocortical network fingerprints differ between essential, Parkinson's and mimicked tremors. Brain. 2018 Jun;141(6):1770-81.

9 Lenka A, Bhalsing KS, Panda R, Jhunjhunwala K, Naduthota RM, Saini J, et al. Role of altered cerebello-thalamo-cortical network in the neurobiology of essential tremor. Neuroradiology. $2017 \mathrm{Feb}$;59(2):157-68.

10 Buijink AW, van der Stouwe AM, Broersma M, Sharifi S, Groot PF, Speelman JD, et al. Motor network disruption in essential trem- or: a functional and effective connectivity study. Brain. 2015 Oct;138(Pt 10):2934-47.

11 Coenen VA, Allert N, Paus S, Kronenbürger M, Urbach H, Mädler B. Modulation of the cerebello-thalamo-cortical network in thalamic deep brain stimulation for tremor: a diffusion tensor imaging study. Neurosurgery. 2014 Dec;75(6):657-69.

12 Hallett M. Tremor: pathophysiology. Parkinsonism Relat Disord. 2014 Jan;20 Suppl 1: S118-22.

13 Bhalsing KS, Saini J, Pal PK. Understanding the pathophysiology of essential tremor through advanced neuroimaging: a review. J Neurol Sci. 2013 Dec;335(1-2):9-13.

14 Tröster AI, Woods SP, Fields JA, Lyons KE, Pahwa R, Higginson CI, et al. Neuropsychological deficits in essential tremor: an expression of cerebello-thalamo-cortical pathophysiology? Eur J Neurol. 2002 Mar;9(2):143-51. 
15 Louis ED. Re-thinking the biology of essential tremor: from models to morphology. Parkinsonism Relat Disord. 2014 Jan;20 Suppl 1: S88-93.

16 Louis ED. Linking Essential Tremor to the Cerebellum: neuropathological Evidence. Cerebellum. 2016 Jun;15(3):235-42.

17 Louis ED, Lee M, Babij R, Ma K, Cortés E, Vonsattel JP, et al. Reduced Purkinje cell dendritic arborization and loss of dendritic spines in essential tremor. Brain. 2014 Dec;137(Pt 12):3142-8

18 Babij R, Lee M, Cortés E, Vonsattel JP, Faust PL, Louis ED. Purkinje cell axonal anatomy: quantifying morphometric changes in essential tremor versus control brains. Brain. 2013 Oct;136(Pt 10):3051-61.

19 Kuo SH, Tang G, Louis ED, Ma K, Babji R Balatbat $\mathrm{M}$, et al. Lingo-1 expression is increased in essential tremor cerebellum and is present in the basket cell pinceau. Acta Neuropathol. 2013 Jun;125(6):879-89.

20 Paris-Robidas S, Brochu E, Sintes M, Emond V, Bousquet M, Vandal M, et al. Defective dentate nucleus GABA receptors in essential tremor. Brain. 2012 Jan;135(Pt 1):105-16.

21 Lin CY, Louis ED, Faust PL, Koeppen AH, Vonsattel JP, Kuo SH. Abnormal climbing fibre-Purkinje cell synaptic connections in the essential tremor cerebellum. Brain. 2014 Dec;137(Pt 12):3149-59.

22 Choe M, Cortés E, Vonsattel JP, Kuo SH, Faust PL, Louis ED. Purkinje cell loss in essential tremor: random sampling quantification and nearest neighbor analysis. Mov Disord. 2016 Mar;31(3):393-401.

23 Louis ED, Faust PL, Vonsattel JP, Honig LS, Rajput A, Robinson CA, et al. Neuropathological changes in essential tremor: 33 cases compared with 21 controls. Brain. 2007 Dec 130(Pt 12):3297-307.

24 Yu M, Ma K, Faust PL, Honig LS, Cortés E, Vonsattel JP, et al. Increased number of Purkinje cell dendritic swellings in essential tremor. Eur J Neurol. 2012 Apr;19(4):625-30.

25 Kuo SH, Erickson-Davis C, Gillman A, Faust PL, Vonsattel JP, Louis ED. Increased number of heterotopic Purkinje cells in essential tremor. J Neurol Neurosurg Psychiatry. 2011 Sep; 82(9): 1038-40

26 Louis ED, Kuo SH, Tate WJ, Kelly GC, Gutierrez J, Cortes EP, et al. Heterotopic Purkinje Cells: a Comparative Postmortem Study of Essential Tremor and Spinocerebellar Ataxias 1, 2, 3, and 6. Cerebellum. 2018 Apr;17(2): 104-10.

27 Delay C, Tremblay C, Brochu E, Paris-Robidas $\mathrm{S}$, Emond V, Rajput AH, et al. Increased LINGO1 in the cerebellum of essential tremor patients. Mov Disord. 2014 Nov;29(13):1637-47.

28 Shill HA, Adler CH, Sabbagh MN, Connor DJ, Caviness JN, Hentz JG, et al. Pathologic findings in prospectively ascertained essential tremor subjects. Neurology. 2008 Apr;70(16 Pt 2):1452-5.

29 Louis ED, Rios E, Rao AK. Tandem gait performance in essential tremor: clinical corre- lates and association with midline tremors. Mov Disord. 2010 Aug;25(11):1633-8.

30 Singer C, Sanchez-Ramos J, Weiner WJ. Gait abnormality in essential tremor. Mov Disord. 1994 Mar;9(2):193-6.

31 Kronenbuerger $\mathrm{M}$, Konczak J, Ziegler W, Buderath P, Frank B, Coenen VA, et al. Balance and motor speech impairment in essential tremor. Cerebellum. 2009 Sep;8(3):38998.

32 Parisi SL, Héroux ME, Culham EG, Norman KE. Functional mobility and postural control in essential tremor. Arch Phys Med Rehabil. 2006 Oct;87(10):1357-64.

33 Filip P, Lungu OV, Bareš M. Dystonia and the cerebellum: a new field of interest in movement disorders? Clin Neurophysiol. 2013 Jul; 124(7):1269-76.

34 Kaji R, Bhatia K, Graybiel AM. Pathogenesis of dystonia: is it of cerebellar or basal ganglia origin? J Neurol Neurosurg Psychiatry. 2018 May;89(5):488-92.

35 Putzke JD, Whaley NR, Baba Y, Wszolek ZK, Uitti RJ. Essential tremor: predictors of disease progression in a clinical cohort. J Neurol Neurosurg Psychiatry. 2006 Nov;77(11): $1235-7$.

36 Louis ED, Agnew A, Gillman A, Gerbin M, Viner AS. Estimating annual rate of decline: prospective, longitudinal data on arm tremor severity in two groups of essential tremor cases. J Neurol Neurosurg Psychiatry. 2011 Jul; 82(7):761-5.

37 Louis ED, Frucht SJ, Rios E. Intention tremor in essential tremor: prevalence and association with disease duration. Mov Disord. 2009 Mar;24(4):626-7.

38 Cohen O, Pullman S, Jurewicz E, Watner D, Louis ED. Rest tremor in patients with essential tremor: prevalence, clinical correlates, and electrophysiologic characteristics. Arch Neurol. 2003 Mar;60(3):405-10.

39 Louis ED, Hernandez N, Michalec M. Prevalence and correlates of rest tremor in essential tremor: cross-sectional survey of 831 patients across four distinct cohorts. Eur J Neurol. 2015 Jun;22(6):927-32.

40 Louis ED, Rios E, Applegate LM, Hernandez NC, Andrews HF. Jaw tremor: prevalence and clinical correlates in three essential tremor case samples. Mov Disord. 2006 Nov;21(11): 1872-8.

41 Louis ED, Gerbin M, Galecki M. Essential tremor 10,20,30, 40: clinical snapshots of the disease by decade of duration. Eur J Neurol. 2013 Jun;20(6):949-54

42 Stolze H, Petersen G, Raethjen J, Wenzelburger R, Deuschl G. The gait disorder of advanced essential tremor. Brain. 2001 Nov;124(Pt 11): 2278-86.

43 Louis ED. Essential Tremor with Head Tremor: trait or State? Can J Neurol Sci. 2016 May; 43(3):443-4.

44 Thawani SP, Schupf N, Louis ED. Essential tremor is associated with dementia: prospective population-based study in New York. Neurology. 2009 Aug;73(8):621-5.
45 Bermejo-Pareja F, Louis ED, Benito-León J; Neurological Disorders in Central Spain (NEDICES) Study Group. Risk of incident dementia in essential tremor: a population-based study. Mov Disord. 2007 Aug;22(11):1573-80.

46 Benito-León J, Louis ED, Mitchell AJ, Bermejo-Pareja F. Elderly-onset essential tremor and mild cognitive impairment: a populationbased study (NEDICES). J Alzheimers Dis. 2011;23(4):727-35

47 Shill HA, Hentz JG, Jacobson SA, Belden C, Sabbagh MN, Beach TG, et al. Essential Tremor in the Elderly and Risk for Dementia. J Neurodegener Dis. 2014;2014:328765.

48 Louis ED, Joyce JL, Cosentino S. Mind the gaps: What we don't know about cognitive impairment in essential tremor. Parkinsonism Relat Disord. 2019 Jun;63:10-19.

49 Louis ED. Non-motor symptoms in essential tremor: A review of the current data and state of the field. Parkinsonism Relat Disord. 2016 Jan;22 Suppl 1:S115-8.

50 Ondo WG, Sutton L, Dat Vuong K, Lai D, Jankovic J. Hearing impairment in essential tremor. Neurology. 2003 Oct;61(8):1093-7.

51 Bhatia KP, Bain P, Bajaj N, Elble RJ, Hallett M, Louis ED, et al. Consensus Statement on the classification of tremors. from the task force on tremor of the International Parkinson and Movement Disorder Society. Mov Disord. 2018 Jan;33(1):75-87.

52 Louis ED. Essential tremor: "Plus" or "Minus". Perhaps now is the time to adopt the term "the essential tremors". Parkinsonism Relat Disord. 2018 Nov;56:111-2.

53 Prasad S, Pal PK. Reclassifying essential tremor: implications for the future of past research. Mov Disord. 2019 Mar;34(3):437.

54 Rajalingam R, Breen DP, Lang AE, Fasano A. Essential tremor plus is more common than essential tremor: insights from the reclassification of a cohort of patients with lower limb tremor. Parkinsonism Relat Disord. 2018 Nov; 56:109-10.

55 Rajalingam R, Breen DP, Chen R, Fox S, Kalia LV, Munhoz RP, et al. The clinical significance of lower limb tremors. Parkinsonism Relat Disord. 2019 Aug;65:165-171.

56 Hopfner F, Deuschl G. Is essential tremor a single entity? Eur J Neurol. 2018 Jan;25(1):71-82.

57 Louis ED, Wendt KJ, Ford B. Senile tremor. What is the prevalence and severity of tremor in older adults? Gerontology. 2000 Jan-Feb; 46(1):12-6.

58 Louis ED. Twelve clinical pearls to help distinguish essential tremor from other tremors. Expert Rev Neurother. 2014 Sep;14(9):1057-65.

59 Zubair A, Cersonsky TEK, Kellner S, Huey ED, Cosentino S, Louis ED. What Predicts Mortality in Essential Tremor? A Prospective, Longitudinal Study of Elders. Front Neurol. 2018 Dec;9:1077.

60 van Egmond ME, Contarino MF, Lugtenberg $\mathrm{CH}$, Peall KJ, Brouwer OF, Fung VS, et al. Variable Interpretation of the Dystonia Consensus Classification Items Compromises Its Solidity. Mov Disord. 2019 Mar;34(3):317-20. 